\title{
Influence of Synchronous Condensers on Operation Characteristics of Double-Infeed LCC-HVDCs
}

\author{
Xu Luo ${ }^{1}$, Fan $\mathrm{Li}^{2}{ }^{2}$, Li Fan ${ }^{1}$, Tao Niu ${ }^{2, *}$, Bo Li $^{1}$, Linxuan Tian ${ }^{2}$ and Hongjie Yu ${ }^{1}$ \\ 1 State Grid Chongqing Economic Research Institute, Chongqing 400044, China; cqkndy@163.com (X.L.); \\ fanli@163.com (L.F.); robertlee1994@126.com (B.L.); yuhongie825@163.com (H.Y.) \\ 2 College of Electrical Engineering, Chongqing University, Chongqing 400044, China; \\ 202011131080t@cqu.edu.cn (F.L.); tlx_cqu_edu@163.com (L.T.) \\ * Correspondence: niutthu@cqu.edu.cn
}

Citation: Luo, X.; Li, F.; Fan, L.; Niu, T.; Li, B.; Tian, L.; Yu, H. Influence of Synchronous Condensers on Operation Characteristics of Double-Infeed LCC-HVDCs. Processes 2021, 9, 1704. https:// doi.org/10.3390/pr9101704

Academic Editors: Sérgio Ramos and João Soares

Received: 2 August 2021

Accepted: 13 September 2021

Published: 23 September 2021

Publisher's Note: MDPI stays neutral with regard to jurisdictional claims in published maps and institutional affiliations.

Copyright: (c) 2021 by the authors. Licensee MDPI, Basel, Switzerland. This article is an open access article distributed under the terms and conditions of the Creative Commons Attribution (CC BY) license (https:/ / creativecommons.org/licenses/by/ $4.0 /)$.

\begin{abstract}
Considering the advantages that dynamic reactive power (var) equipment (such as synchronous condensers (SCs), which can control var independently and improve voltage stability), SCs are widely used in AC/DC hybrid power grid to provide emergency var and voltage support. In order to evaluate the dynamic var reserve capacity of SCs and analyze the influence of SCs on the operation characteristics of power system, a model with double-infeed line-commutated converter-based high-voltage direct currents (LCC-HVDCs) and SCs is established. Through theoretical derivation and PSCAD/EMTDC simulation, the effects of SCs on the operation characteristics of double-infeed LCC-HVDCs networks are studied. Then, the non-smooth voltage waveform of electromagnetic transient simulation is approximately transformed into smooth waveform by data fitting method. Finally, the processed voltage waveform is searched step by step to explore the boundary of voltage safety region to determine the dynamic var reserve capacity of SCs. The numerical results show that SCs can enlarge the voltage security region of the direct current (DC) subsystem, thus effectively improving the steady-state and transient security level of the double-infeed LCC-HVDCs networks.
\end{abstract}

Keywords: synchronous condensers (SCs); var reserve capacity; double-infeed LCC-HVDCs; electromagnetic transient simulation; data fitting method

\section{Introduction}

In China, the renewable energy represented by wind energy and photovoltaic is mainly distributed in the western region of China, while the load is mainly distributed in the southeast region. As the channel of energy transmission, LCC-HVDCs can significantly improve the utilization efficiency and consumption level of renewable energy. LCC-HVDCs has obvious advantages in operation stability, transmission capacity, and transmission distance, and thus it plays an important role in the selection of energy transmission mode. In East and Southeast China, power grid structure with multi-infeed LCC-HVDCs has been formed. Due to the heavy load density at the receiving end, the electrical distances between some DC dropping points are relatively close, and therefore the stability of the system has been greatly challenged.

Since thyristors does not have the ability of self-closed thyristors, which are used as the commutation elements, LCC-HVDCs often occur commutation failures due to alternating current $(\mathrm{AC})$ system faults at the receiving end. In China, when three continuous commutation failures occur, protection of DC blocking will work. For example, in September 2015, commutation failures caused by AC line faults have occurred in East China power grid. The bipolar DC blocking of Jinsu HVDC network occurred, and the loss of power grid was $4.90 \mathrm{GW}$ and the lowest frequency dropped to $49.85 \mathrm{~Hz}$, which seriously worsened the safe operation level of the power grid.

When commutation failures occur, the var required by the converter station at the receiving end increases sharply, and the bus voltage at the inverter side decreases sharply 
at the same time, which leads to a significant reduction in the var support provided by capacitors in the system. The lack of var support will cause the expansion of the fault and the collapse of voltage in serious cases. Many measures have been taken to prevent commutation failures. The mechanism and influencing factors of commutation failure were analyzed in detail in the literature [1,2], and the main cause of commutation failure was summarized as a too small extinction angle under the operation state. In order to effectively improve the safe operation level, the power grid topology was improved to carry out the commutation failure predictive control [3]; a new topology based on fixed capacitor and controllable capacitor commutation converter is proposed in [4]; another widely used measure is adding dynamic var compensation equipment such as static var compensator (SVC), static var generator (SVG), and synchronous condensers at the receiving end $[5,6]$, which can meet the var demand of converter station and improve the voltage recovery speed; some valuable studies calculate the voltage security region to guarantee the voltage stability margin under $\mathrm{N}-1$ faults [7-9] proposes a novel method to discriminate the critical commutation failure region of multi-infeed HVDCs system and the critical failure impedance boundaries on topology diagram can be therefore delimited by examining each bus in the AC system; some other valuable works in the literature $[10,11]$ have studied the influence of electrical distance on the stability of LCC-HVDC. The above research greatly improves the stability and security of power system operation and the commutation failure is effectively restrained.

From the perspective of analytical tools, electromagnetic transient simulation (EGTS) [12] and electromechanical transient simulation (EHTS) are most commonly used [13]. The former is limited by the speed and scale of simulation, while the latter is seriously inaccurate due to the neglect of negative sequence components. When the fault type is asymmetric, it will produce great errors, resulting in more or less dynamic var reserve capacity obtained by simulation. When the value of dynamic var reserve is small, it is difficult to meet the var demand of the system under fault, resulting in the further expansion of the fault range; when the value of dynamic var reserve is too large, due to the high cost of dynamic var equipment, it will bring great economic waste. Intrinsically, obtaining dynamic var capacity of SCs in hybrid AC/DC networks is the computation of optimal power flow considering the constraints of transient stability (TSCOPF). In [14], the trajectory sensitivity analysis is introduced to simplify and solve TSCOPF problems. As shown in Table 1, many scholars have used different methods to solve it.

Table 1. Review of existing research methods.

\begin{tabular}{|c|c|}
\hline References & Method Adopted in References \\
\hline [15] & $\begin{array}{l}\text { Based on the reduced-space interior point method (RIPM)-which takes advantage of the } \\
\text { relatively few degrees of freedom and shows promising potential for solving large-scale } \\
\text { TSCOPF problems-this paper introduces a parallel RIPM algorithm with high computing } \\
\text { efficiency for multi-contingency TSCOPF problems. }\end{array}$ \\
\hline [16] & $\begin{array}{l}\text { In this paper, the constraint of TSCOPF is derived from dynamic information provided } \\
\text { by the Single Machine Equivalent (SIME) method and is only expressed in terms of } \\
\text { steady-state variables, which allows us to diminish the length of the time-domain } \\
\text { simulation to be included into the global TSC-OPF to a single (initial) time step. }\end{array}$ \\
\hline [17] & $\begin{array}{c}\text { A new GSO-PE approach, consisting of an improved group search optimization (GSO) } \\
\text { and } 2 \mathrm{~m}+1 \text { point estimated (PE) method with Cholesky decomposition, is then } \\
\text { designed to effectively solve this challenging P-TSCOPF problem. }\end{array}$ \\
\hline [18] & $\begin{array}{l}\text { This paper presents a novel GPU acceleration approach for transient } \\
\text { stability-constrained optimal power flow (TSCOPF), which is one of the most } \\
\text { computational challenging tasks in large-scale power system applications. Enabled by } \\
\text { the revealed two-level decomposition parallelism in reduced-space interior point } \\
\text { method, GPUs serve as plug-and-play coprocessors for time-consuming linear algebra } \\
\text { operations in TSCOPF solving. }\end{array}$ \\
\hline
\end{tabular}

However, some challenges still remain.

(1) The differential algebraic equations describing the transient characteristics of hybrid AC/DC power grid have strong rigidity [19], which is reflected by waveform in 
the simulation, resulting in dramatic changes and many burrs in the simulation waveform; when the step searching algorithm is used to determine the voltage security region boundary, a large error will occur.

(2) The large-scale TSCOPF problem has the characteristics of high nonlinearity and dimension, which also contains integer variables. How to efficiently solve large-scale TSCOPF problem is still a problem. The traditional method to solve TSCOPF problem is trajectory sensitivity analysis. However, due to the large scale of hybrid AC/DC power grid, it is difficult to realize the online solution considering that the dispatching time scale of China power grid is generally $5-15 \mathrm{~min}$ [20-22].

(3) Since the DC subsystem has complex control links, the state equations describing these control links are often discontinuous, which will lead to state jump or nonconvergence.

Therefore, in order to solve these challenges, a novel method to obtain dynamic var reserve in hybrid AC/DC networks is presented in this paper. The main contribution of this paper is listed as follows:

(1) The voltage sag area under fault is selected as the index to judge the fault severity, and the influence of different short circuit ratio and electrical distance of HVDC subsystem on voltage are analyzed clearly;

(2) With the help of data fitting method, the original voltage waveform with strong rigidity and many burrs is replaced by an approximately smooth voltage curve, and then the voltage stability boundary and dynamic var reserve capacity are determined by the step search algorithm. The proposed method is efficient and feasible, and is more convenient to be applied in engineering practice compared with traditional TSCOPF solving methods.

The structure of this paper is as follows: in Section 2, a model with double-infeed LCCHVDCs system is established and detailed parameters are introduced. Then in Section 2.2, the mathematical model of double-infeed LCC-HVDCs is derived and the influence of DC subsystem on power system operation is analyzed. In Section 3, the voltage sag area is introduced to quantify the severity of the faults. Next, the step searching algorithm and data fitting method are adopted to obtain dynamic var reserve capacity of SCs in Section 3.2. In the case study, the accuracy and efficiency of the method proposed in this paper are verified. Finally, Section 5 summarizes the full paper and gives the potential research directions in the future.

\section{Double-Infeed LCC-HVDC System Model with Synchronous Condenser}

2.1. The Introduction of the General Model

The general model of double-infeed HVDC system with SC is shown as Figure 1.

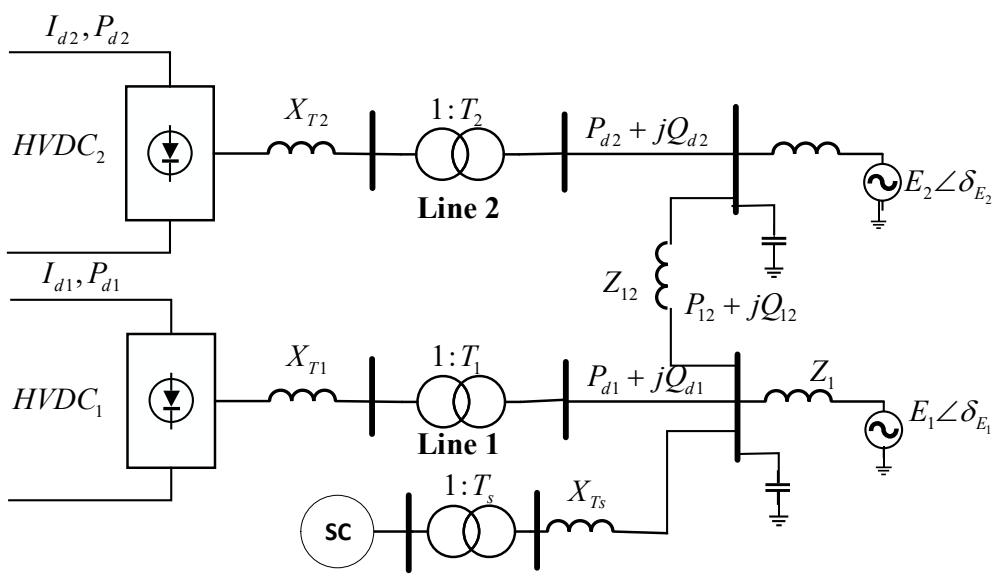

Figure 1. Model of double-infeed LCC-HVDCs with SCs.

The control modes of the first HVDC subsystem $\left(\mathrm{HVDC}_{1}\right)$ and the second HVDC subsystem $\left(\mathrm{HVDC}_{2}\right)$ are constant current control and minimum trigger angle control at 
the rectifier side and constant extinction angle control and constant current control at the inverter side.

It should be noted that the parameters of the model are the same with the CIGRE standard model and the capacity is $1000 \mathrm{MW}$. SC is connected to line-1. HVDC 1 is connected to $\mathrm{HVDC}_{2}$ through line $\mathrm{Z}_{12}$, which is seen as the index reflecting the electrical distance. The relevant parameters of DC subsystem are set according to CIGRE model, which will not be described in detail here.

\subsection{The Power Transmission Mathematical Model of the Double-Infeed LCC-HVDCs with Synchronous Condenser}

For the independent $\mathrm{HVDC}_{1}$ system, the mathematical model can be described as Equations (1)-(10).

$$
\begin{gathered}
I_{d 1}=\frac{U_{1}\left[\cos \gamma_{1}-\cos \left(\gamma_{1}+\mu_{1}\right)\right]}{\sqrt{2} T_{1} X_{T 1}} \\
U_{d 1}=\frac{3 \sqrt{2} U_{1}}{\pi T_{1}} \cos \gamma_{1}-\frac{3}{\pi} X_{T 1} I_{d 1} \\
P_{d 1}=U_{d 1} I_{d 1} \\
Q_{d 1}=P_{d 1} \tan \phi_{1} \\
\cos \phi_{1}=-\frac{\cos \gamma_{1}+\cos \left(\gamma_{1}+\mu_{1}\right)}{2} \\
P_{a c 1}=\left[U_{1}^{2} \cos \theta_{1}-E_{1} U_{1} \cos \left(\delta_{U 1}-\delta_{E 1}+\theta_{1}\right)\right] /\left|Z_{1}\right| \\
Q_{a c 1}=\left[U_{1}^{2} \sin \theta_{1}-E_{1} U_{1} \sin \left(\delta_{U 1}-\delta_{E 1}+\theta_{1}\right)\right] /\left|Z_{1}\right| \\
Q_{c 1}=B_{c 1} U_{1}^{2} \\
P_{d 1}-P_{a c 1}-P_{c 1}=0 \\
Q_{d 1}-Q_{a c 1}+Q_{c 1}=0
\end{gathered}
$$

Here, $U_{d 1}$ and $I_{d 1}$ represent DC voltage and current; $P_{d 1}$ and $Q_{d 1}$ represent active power and reactive power at the DC side, respectively. $P_{a c 1}$ and $Q_{a c 1}$ represent active power and reactive power at $\mathrm{AC}$ side, respectively. $P_{c 1}$ and $Q_{c 1}$ represent active power loss and var compensation capacity of filters and other var compensation device at the inverter side. $X_{T 1}$ represents leakage reactance of converter transformer and $T_{1}$ represent transformer taps; $B_{c 1}$ represents equivalent susceptance of filters and reactive power compensation device at the converter side; $Z_{1}$ represents equivalent impedance of the system; $U_{1} \angle \delta_{U 1}$ and $E_{1} \angle \delta_{E 1}$ represent $\mathrm{AC}$ bus voltage and $\mathrm{AC}$ electromotive force, respectively. $\mu_{1}$ represents commutation overlap angle and $\gamma_{1}$ represents the turn-off angle. Equations (1)-(10) are derived and mainly used to explain the impact of dynamic var equipment on the power flow in hybrid AC/DC power grid.

The power transmission equation of double-infeed LCC-HVDCs system with synchronous condenser needs to consider the reactive power transmission between synchronous condenser and $\mathrm{HVDC}_{1}$, as well as the active power and reactive power transmission between $\mathrm{HVDC}_{1}$ and $\mathrm{HVDC}_{2}$. Therefore, the power equation of $\mathrm{HVDC}_{1}$ Equations (9) and (10) is modified to Equations (11) and (12).

$$
\begin{gathered}
P_{d 1}-P_{a c 1}-P_{c 1}-P_{12}=0 \\
Q_{d 1}-Q_{a c 1}+Q_{c 1}-Q_{12}+Q_{s}=0
\end{gathered}
$$

Here, $P_{12}$ and $Q_{12}$ are the active power and reactive power transmitted from $H_{V D C}$ to $\mathrm{HVDC}_{2}$ on the connecting line respectively. Qs represents reactive power transmitting from synchronous condenser to $\mathrm{HVDC}_{1}$.

If $Q_{\mathrm{s}}$ is within the capacity range of $\mathrm{SC}$, the $\mathrm{SC}$ absorbs or produces var according to var demand; if $Q_{s}$ is out of the capacity range of SC, then the SC is treated as a constant current 
source. Similarly, the power equations of $\mathrm{HVDC}_{2}$ are shown as Equations (12)-(14). Here, $\Delta P_{12}$ and $\Delta Q_{12}$ represent active power/reactive power loss of tie lines. The expression of power transmission from $\mathrm{HVDC}_{1}$ to $\mathrm{HVDC}_{2}$ is shown as Equation (15).

$$
\begin{aligned}
& P_{d 2}-P_{a c 2}-P_{c 2}+P_{12}-\Delta P_{12}=0 \\
& Q_{d 2}-Q_{a c 2}+Q_{c 2}+Q_{12}-\Delta Q_{12}=0 \\
& P_{12}+j Q_{12}=\dot{U}_{1}\left(\frac{\dot{U}_{1}-\dot{U}_{2}}{Z_{12}}\right)^{*} \\
& \left\{\begin{array}{l}
\frac{d \delta}{d t}=(\omega-1) \omega_{s} \\
\frac{d \omega}{d t}=\frac{1}{T_{j}}\left(-P_{e}-D \omega\right) \\
\frac{d E_{q}^{\prime}}{d t}=\frac{1}{T_{d 0}^{\prime}}\left(-E_{q}^{\prime}-\left(X_{d}-X_{d}^{\prime}\right) i_{d}+E_{f q}\right)
\end{array}\right. \\
& \left\{\begin{array}{c}
E_{q}^{\prime}=v_{q}+R_{a} i_{q}+X_{d}^{\prime} i_{d} \\
0=v_{d}+R_{a} i_{d}-X_{q} i_{q}
\end{array}\right.
\end{aligned}
$$

The mathematical model of SCs can be expressed by Equations (16) and (17). Here, $w$ represents rotor angular speed, $T_{j}$ represents rotor inertia time constant, $P_{e}$ represents electromagnetic power, $D$ represents damping coefficient, $E_{q}{ }^{\prime}$ represents transient electromotive force, $T_{d 0^{\prime}}$ represents direct axis transient open circuit time constant, $X_{d}$ and $X_{q}$ are synchronous reactances of direct axis and quadrature axis respectively, $X_{d}{ }^{\prime}$ represents direct axis transient reactance, $R_{a}$ represents stator resistance, $v_{d}$ and $v_{q}$ represent direct and quadrature axis voltage components, respectively, $i_{d}$ and $i_{q}$ represent direct axis and quadrature axis current components, respectively, and $E_{f q}$ represents excitation voltage.

It is widely accepted that SCs can provide fast var support when fault occurs in the hybrid AC/DC system. When the bus voltage of inverter converter station drops sharply, the var increment of the SCs is as follows:

$$
\begin{aligned}
& \Delta Q=Q-Q_{0}=U_{L} i_{d}-U_{0} i_{d 0} \approx \\
& \left(U_{0}+\Delta U\right)\left(i_{d 0}+\Delta i_{d}\right)-U_{0} i_{d 0}=U_{L} \Delta i_{d}+\Delta U i_{d 0} .
\end{aligned}
$$

Here, $\Delta U$ and $U_{L}$ represents the increment of bus voltage and bus voltage after short circuit fault, respectively; $\Delta i_{d}$ and $i_{d 0}{ }^{\prime}$ represent reactive current increment of synchronous condenser's D-axis and reactive current of D-axis before fault, respectively.

SC is essentially a no-load synchronous generator. Its function is to provide var for the system. The var can be regulated by adjusting the excitation current of SC. During over-excitation operation, the SC sends out inductive var and the system voltage increases. During under-excitation operation, the SC sends out capacitive var and the system voltage decreases. As shown in the Figure 2 below, because SCs have the ability to continuously adjust var, it can be used to improve voltage recovery speed and prevent continuous commutation failure.

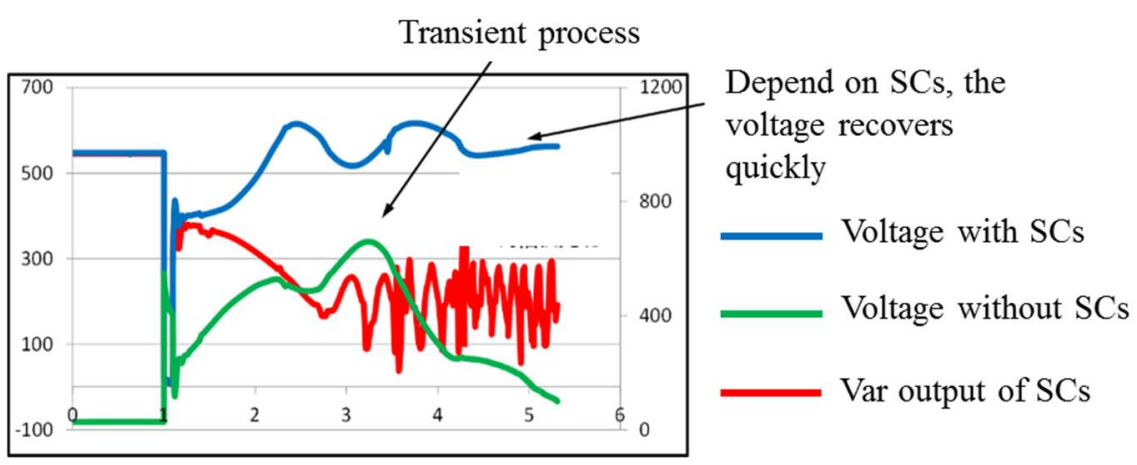

Figure 2. Performance comparison with/without SCs inclusion. 
Although the multi-infeed LCC-HVDCs increase the capacity of the system at the receiving end, it also increases the spread path of voltage collapse under N-1 faults. SCs can quickly release a large amount of var in case of fault, providing emergent var and voltage support for the system. The spontaneous var response of SCs is an important means to suppress consecutive commutation failure.

\section{Operational Risk Analysis of Multi-Infeed LCC-HVDCs}

\subsection{Multi-Infeed LCC-HVDCs Voltage Sag Risk Assessment Method}

In this section, the voltage sag area under fault is chosen as the index of voltage safety margin, and the index of voltage safety margin under different short circuit ratio (SCR) and different electrical distance between LCC-HVDC subsystems is calculated. The test system is built in PSCAD/EMTDC and the fault type is three-phase grounding fault. The fault occurrence time is $1 \mathrm{~s}$. The fault clearing time is $1.2 \mathrm{~s}$, and the grounding resistance is $0.1 \mathrm{ohm}$.

Figure 3 shows the calculation method of voltage drop area. Obviously, as shown in Table 2, the larger the short circuit ratio, the smaller the corresponding voltage sag area, which indicates that the stronger the AC system supports the transient voltage under the $\mathrm{N}-1$ condition. On the other hand, the electrical distance between the receiving end will also affect the final result of voltage safety margin. When the electrical distance between subsystems is small, this indicates that the electrical coupling strength of the system is great, and the fault influence range is wide. Therefore, when analyzing the operation state of multi-infeed LCC-HVDCs, it is necessary to consider the coupling relationship between HVDC subsystems.

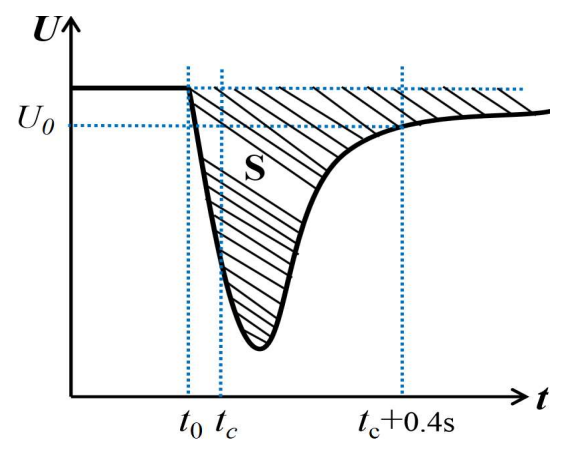

Figure 3. Schematic diagram of voltage sag area.

Table 2. Calculation results of voltage sag area under different conditions.

\begin{tabular}{ccccc}
\hline Electrical Distance & \multicolumn{2}{c}{ Short Circuit Ratio } & \multicolumn{2}{c}{ Voltage Sag Area } \\
\hline \multirow{2}{*}{$\mathrm{Z}=50+\mathrm{j} 0.2$} & $\mathrm{SCR}_{1}=2.5$ & $\mathrm{SCR}_{2}=2.5$ & $\mathrm{~S}_{1}=0.9741$ & $\mathrm{~S}_{2}=0.6812$ \\
& $\mathrm{SCR}_{1}=2.5$ & $\mathrm{SCR}_{2}=4$ & $\mathrm{~S}_{1}=0.8477$ & $\mathrm{~S}_{2}=0.6385$ \\
& $\mathrm{SCR}_{1}=4$ & $\mathrm{SCR}_{2}=4$ & $\mathrm{~S}_{1}=0.7602$ & $\mathrm{~S}_{2}=0.7724$ \\
\hline \multirow{3}{*}{$\mathrm{Z}=100+\mathrm{j} 0.4$} & $\mathrm{SCR}_{1}=2.5$ & $\mathrm{SCR}_{2}=2.5$ & $\mathrm{~S}_{1}=0.8362$ & $\mathrm{~S}_{2}=0.7837$ \\
& $\mathrm{SCR}_{1}=4$ & $\mathrm{SCR}_{2}=2.5$ & $\mathrm{~S}_{1}=0.6900$ & $\mathrm{~S}_{2}=0.5293$ \\
& $\mathrm{SCR}_{1}=4$ & $\mathrm{SCR}_{2}=4$ & $\mathrm{~S}_{1}=0.7594$ & $\mathrm{~S}_{2}=0.4982$ \\
\hline
\end{tabular}

\subsection{Calculation Method of Var Reserve Capacity of Synchronous Condenser}

The traditional method to calculate the dynamic var reserve capacity under transient conditions is to solve the TSCOPF problem, and the existence of differential equations describing transient process greatly increases the difficulty of problem-solving. In the electromagnetic transient simulation, the waveform is not smooth and full of burr, which leads to a large error using step searching algorithm to obtain the boundary of voltage security region. Therefore, this paper uses data fitting method to replace the complicated and burry electromagnetic transient simulation curve with smooth curve, which can be 
described accurately by high-order polynomial. In addition, in order to use the step searching algorithm more conveniently, it is necessary to calculate the sensitivity coefficient of the var output of the synchronous condenser to the bus voltage.

Figure 4 shows the calculation diagram of var reserve capacity. Considering the differential equations describing the dynamic characteristics of converter station have the disadvantages such as high order, strong rigidity and time-varying, therefore, it is difficult to analyze and deal with them directly. The data fitting method skips over the complex state equation and topological structure of converter station, and focuses on the historical operation data to fit the algebraic relationship between voltage amplitude and many operation parameters including reactive power, active power, voltage, current of converter station, var output of SCs, fault occurrence time, duration time and grounding impedance, which can obviously reduce the computational complexity, obtain accurate results and improve the solution speed.

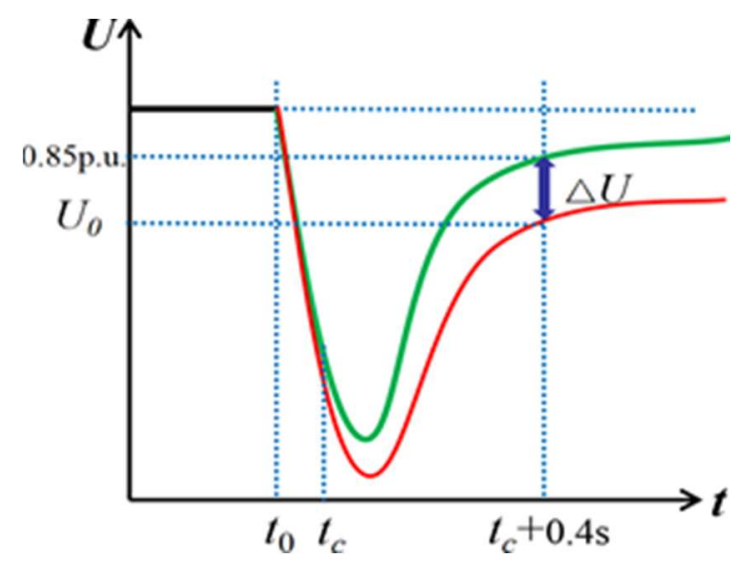

Figure 4. Calculation diagram of var reserve capacity.

The most commonly used fitting method is the least square method. The mathematical expression of the traditional least square method is as follows in Equations (19)-(21):

$$
\begin{aligned}
& \mathbf{X}=\left[\begin{array}{ccccc}
1 & x_{1}^{(1)} & x_{2}^{(1)} & \cdots & x_{k}^{(1)} \\
1 & x_{1}^{(2)} & x_{2}^{(2)} & \cdots & x_{k}^{(2)} \\
1 & \vdots & \vdots & \vdots & \vdots \\
1 & x_{1}^{(N)} & x_{2}^{(N)} & \cdots & x_{k}^{(N)}
\end{array}\right]_{N \times(k+1)} \\
& \boldsymbol{\alpha}=\left[\begin{array}{lllllll}
a_{0} & a_{1} & a_{2} & \ldots & a_{k}
\end{array}\right]_{1 \times(k+1)}^{T} \mathbf{Y}=\left[\begin{array}{lllll}
y_{1} & y_{2} & y_{3} & \cdots & y_{N}
\end{array}\right]_{1 \times N}^{T} \mathbf{Y}=\mathbf{X} \boldsymbol{\alpha} \\
& \varepsilon=\|\mathbf{Y}-\mathbf{X} \boldsymbol{\alpha}\|_{2}^{2}=(\mathbf{Y}-\mathbf{X} \boldsymbol{\alpha})^{T}(\mathbf{Y}-\mathbf{X} \boldsymbol{\alpha}) \Rightarrow \min
\end{aligned}
$$

However, the least square method still has its limitations. It is sensitive to the sampling points of data outliers. For the least square method fitting of multivariate unknown parameters, iterative solution is needed in the process of parameter fitting, and the appropriate gradient descent method needs to be selected, which may encounter the situation that the inverse matrix does not exist or the initial value is too far from the local minimum.

$$
\begin{aligned}
& \gamma=\sum_{i=1}^{m} A_{m} P_{n}{ }^{m}+\sum_{i=1}^{m} B_{m} Q_{n}{ }^{m}+ \\
& \sum_{i=1}^{m} C_{m} U_{n}{ }^{m}+\sum_{i=1}^{m} D_{m} I_{n}{ }^{m}+\sum_{i=1}^{m} E_{m} Q_{s c n}{ }^{m} \\
& +\sum_{i=1}^{m} F_{m} R_{n}{ }^{m}+G
\end{aligned}
$$




$$
\begin{aligned}
& Y=\left[y_{1}, y_{2}, y_{3} \cdots \cdots y_{n}\right]^{T} \\
& X=\left[A_{1} \cdots A_{m}, B_{1} \cdots B_{m}, \cdots G_{1} \cdots G_{m}\right]^{T} \\
& H=\left[\begin{array}{cccc}
P_{n}^{1} & Q_{n}^{1} & \cdots & R_{n}^{1} \\
P_{n}^{2} & Q_{n}^{2} & \cdots & R_{n}^{2} \\
\cdots & \cdots & \cdots & \cdots \\
P_{n}^{m} & Q_{n}^{m} & \cdots & R_{n}^{m}
\end{array}\right] \\
& \|E\|=Y-H X=\sum_{i=1}^{m} e_{1}^{2} \leq \varepsilon
\end{aligned}
$$

Thus, in this paper, as shown in Equations (22) and (23), the fitting method based on improved Powell algorithm is used to fit and analyze the operation data of hybrid AC/DC power system. The improved Powell optimization algorithm is a search algorithm based on the property that conjugate direction can speed up the convergence speed. It can effectively improve the convergence speed of fitting and reduce the sensitivity of fitting curve to abnormal data in sampling points on the premise of ensuring the accuracy of fitting. Detailed analysis is discussed below.

The traditional Powell algorithm can be used to solve unconstrained optimization problems of positive definite quadratic functions, as shown in Equation (24).

$$
\begin{aligned}
& \min f(x)=\frac{1}{2} x^{T} Q x+b^{T} x+c \\
& x \in \mathbb{R}^{n}, Q \in \mathbb{R}^{n * n}, b \in \mathbb{R}^{n}, c \in \mathbb{R}
\end{aligned}
$$

The traditional Powell algorithm is essentially a conjugate direction method, since it only needs to calculate the value of the objective function and does not need to seek the derivative value. Therefore, Powell algorithm is more practical than the common conjugate direction method. The optimal solution of the optimization problem is obtained by $n$ times of direction correction and iterative calculation. However, as shown in Figure 5 below, it is not difficult to find that in the traditional Powell algorithm, the $n$ search directions in each iteration must be kept linearly independent, otherwise the optimal solution of the problem will hardly be obtained. When the search directions are linearly correlated, degenerate, or ill-conditioned, the search process is carried out in the reduced dimension space, which makes the calculation unable to converge.

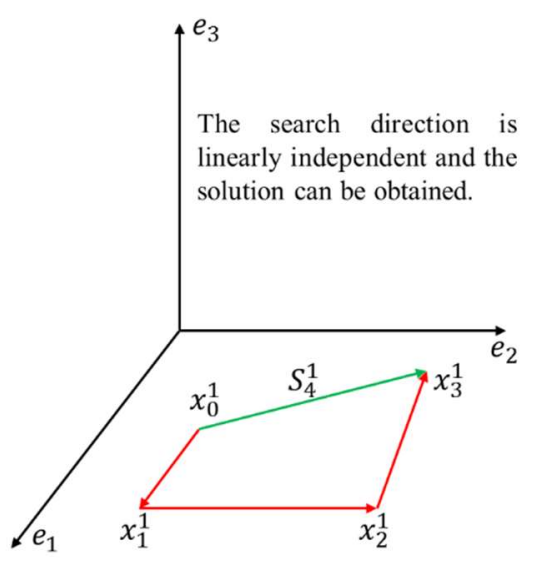

(a) Application scenarios of Powell algorithm

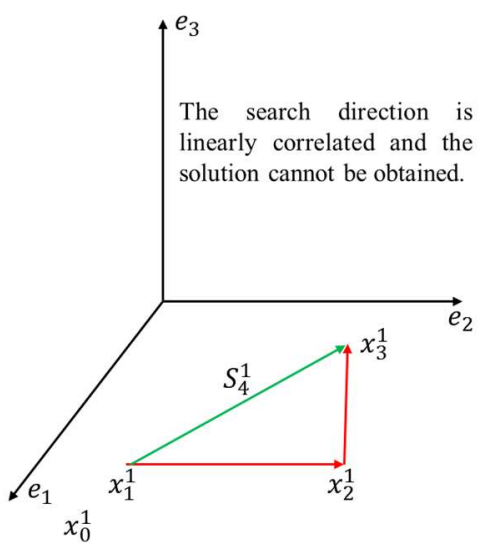

(b) Inapplicable scenarios of Powell algorithm

Figure 5. Characteristic analysis of traditional Powell algorithm.

For the convenience of explanation, we first make the following definition as shown in Equation (25). The meaning of the parameters is clearly shown in Figure 6.

$$
\begin{aligned}
& f_{1}=f\left(x_{k}^{0}\right) \\
& f_{2}=f\left(x_{k}^{n}\right) \\
& f_{3}=f\left(x_{k}^{n+2}\right)
\end{aligned}
$$




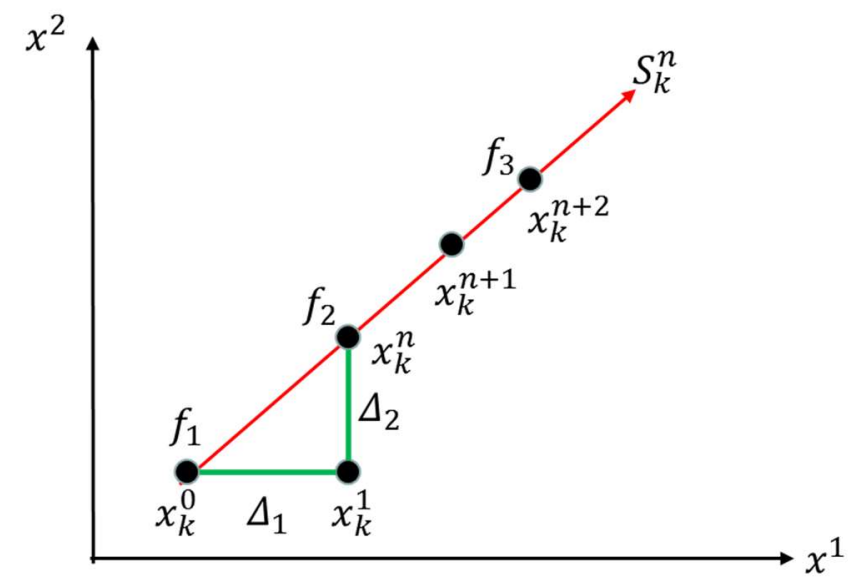

Figure 6. Schematic diagram of parameter description.

Compared with the traditional Powell algorithm, the main improvements of the modified Powell algorithm are listed as follows:

1. Firstly, when forming the $K+1$ direction group, instead of eliminating the first direction in the previous cycle, the function value is calculated and determined according to whether the conditions are met.

2. Secondly, find out the direction $m$ in which the function value decreases the most in the previous iteration and the decrease amount is represented by $\Delta m$, as shown in Equation (26).

$$
\Delta m=\max \left(f\left(x_{k}^{i}\right)-f\left(x_{k}^{i+1}\right)\right), i=0,1, \ldots, n-1
$$

Then, the whole process of improved Powell algorithm is summarized, as shown in Algorithm 1.

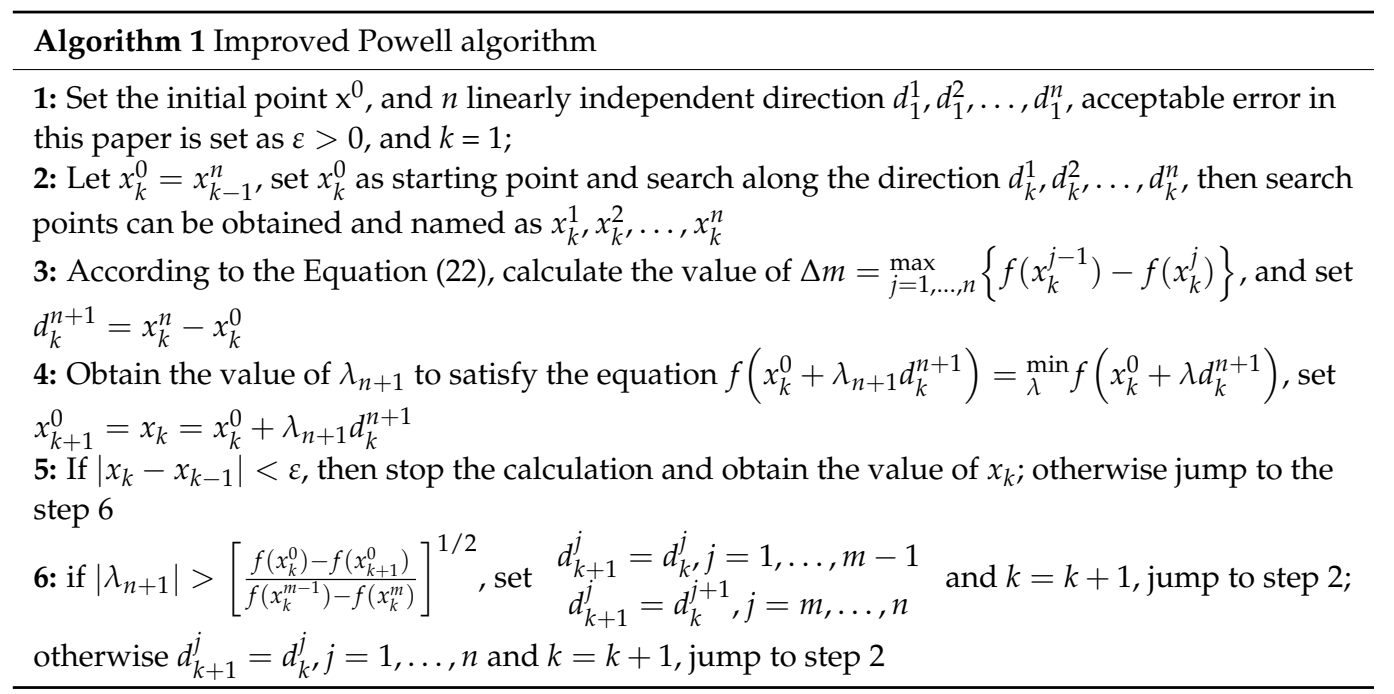

The above is the whole content of data fitting using improved Powell algorithm. Next, when the var output of SCs increases $\Delta \mathrm{Q}$, the corresponding voltage also increases $\Delta \mathrm{U}$, then the sensitivity coefficient $\mathrm{K}$ can be calculated by the following formula:

$$
K=\Delta \mathrm{U} / \Delta \mathrm{Q}
$$

According to the actual needs of the engineering, when the value of voltage restores to 0.85 p.u. in $0.4 \mathrm{~s}$ after the clearing the fault [12], then the continuous commutation failure 
will not occur. Thus, this voltage value is taken as the initial value of step search, and $\Delta \mathrm{Q}$ is accumulated gradually until the following conditions are met:

$$
\mathrm{U}_{0}+K^{*} \Delta \mathrm{Q} \geq 0.85 \text { p.u. }
$$

At this time, the dynamic var reserve capacity is the minimum var reserve capacity to meet the system safety operation and resist $\mathrm{N}-1$ fault in the current scenario. To gain a better understanding, the whole calculation process of dynamic var reserve capacity is shown in Algorithm 2.

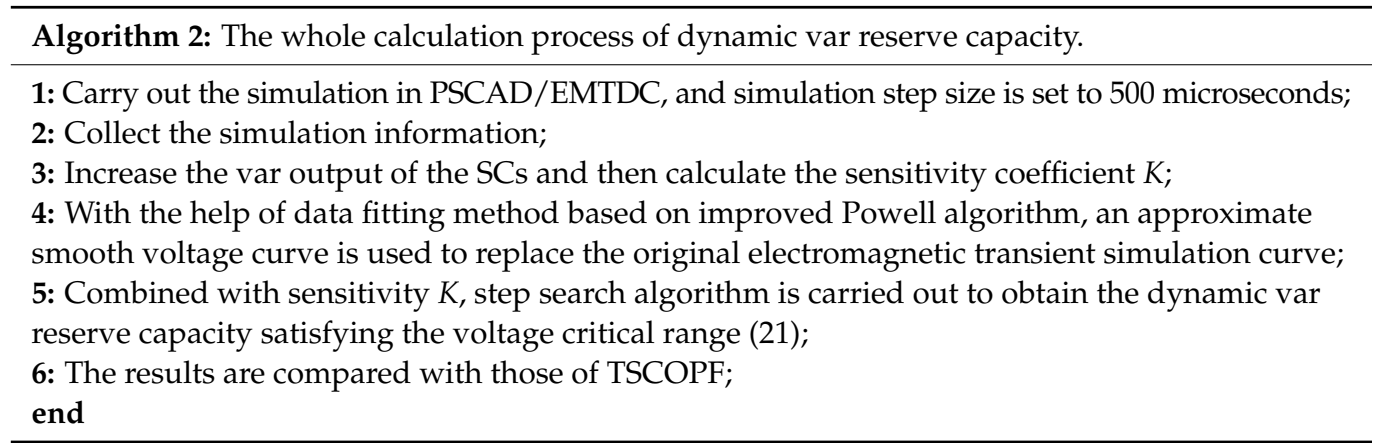

\section{Case Study}

\subsection{The Results of Dynamic Var Reserve Assessment}

In this section, the accuracy and feasibility of the proposed method are illustrated by cases under two different conditions, i.e., single-phase grounding fault and three-phase grounding fault. The model topology used in the cases is shown in Figure 1, and the detailed information of the fault is shown in Table 3.

Table 3. Detailed fault information and results of dynamic var reserve assessment in case study.

\begin{tabular}{ccc}
\hline Information & $\begin{array}{c}\text { Single-Phase Grounding } \\
\text { Fault }\end{array}$ & $\begin{array}{c}\text { Three-Phase Grounding } \\
\text { Fault }\end{array}$ \\
\hline Occurrence time & $4.4 \mathrm{~s}$ & $4.4 \mathrm{~s}$ \\
Clearing time & $4.6 \mathrm{~s}$ & $4.6 \mathrm{~s}$ \\
Grounding resistance & $0.1 \Omega$ & $0.1 \Omega$ \\
\hline $\begin{array}{c}\text { Dynamic var reserve capacity } \\
\text { obtained by the proposed } \\
\text { method/MVAR }\end{array}$ & 34.07 & 68.54 \\
\hline $\begin{array}{c}\text { Dynamic var reserve capacity } \\
\text { obtained by solving } \\
\text { TSCOPF/MVAR }\end{array}$ & 35.26278 & 71.3560 \\
\hline $\begin{array}{c}\text { Bus voltage at tc }=0.4 \mathrm{~s} \text { before } \\
\text { optimization/p.u }\end{array}$ & 0.8480 & 0.8478 \\
\hline Bus voltage at tc $=0.4 \mathrm{~s}$ after \\
optimization/p.u
\end{tabular}

Figure 7a shows the voltage waveform curve obtained by the fitting method based on improved Powell algorithm. The critical value of dynamic var reserve can be obtained by step searching algorithm. Figure $7 \mathrm{c}$,d shows the voltage results after var optimization under different faults. Obviously, after the dynamic var reserve optimization, the bus voltage quickly recovers to 0.85 p.u. after the fault is cleared, which effectively inhibits the occurrence of continuous commutation failure. 


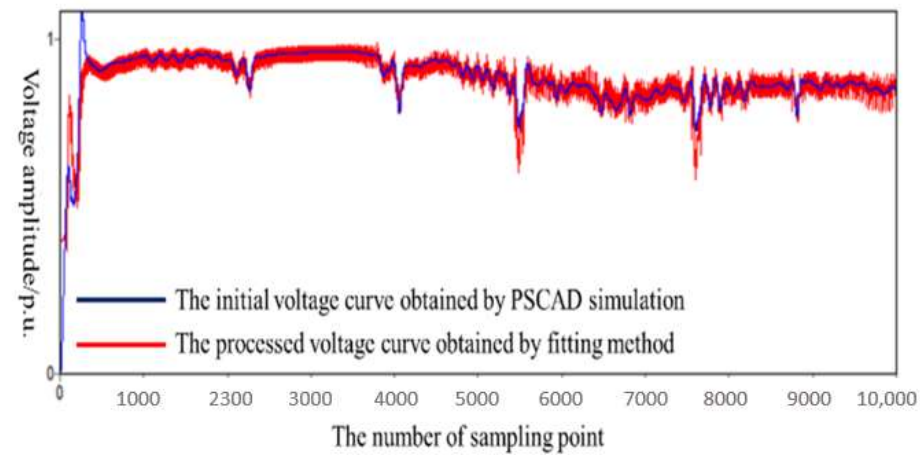

(a) The fitting result of improved Powell algorithm

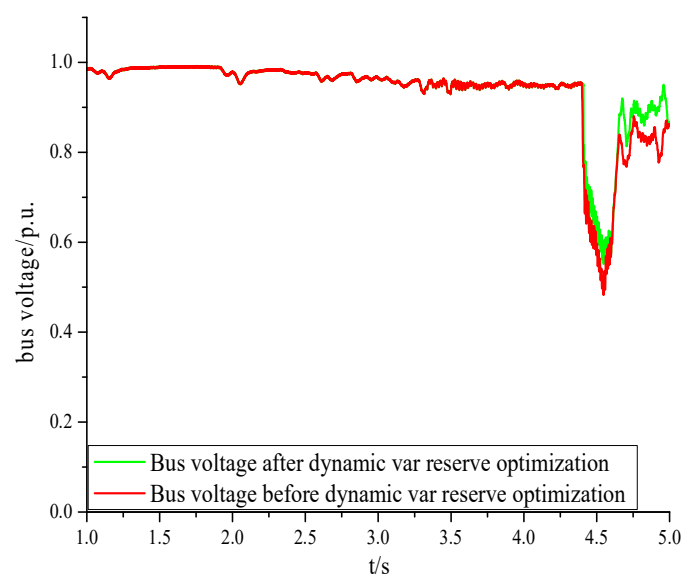

(c) single-phase grounding fault

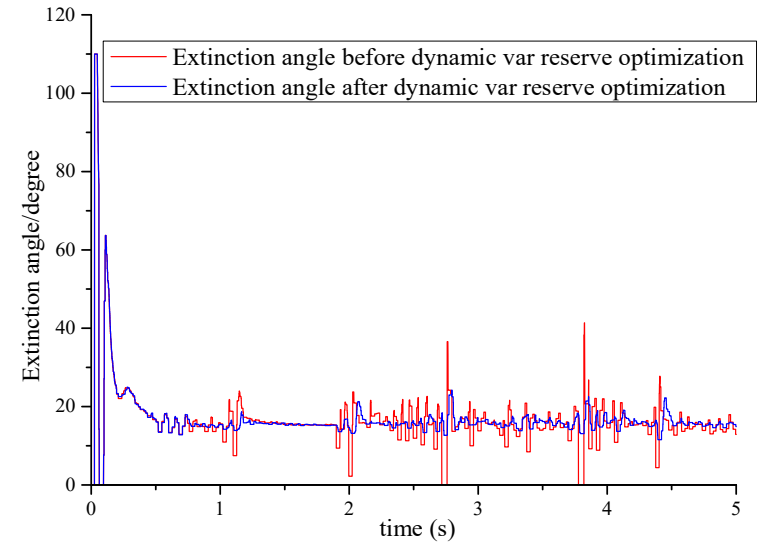

(b) Extinction angle before and after dynamic var reserve optimization

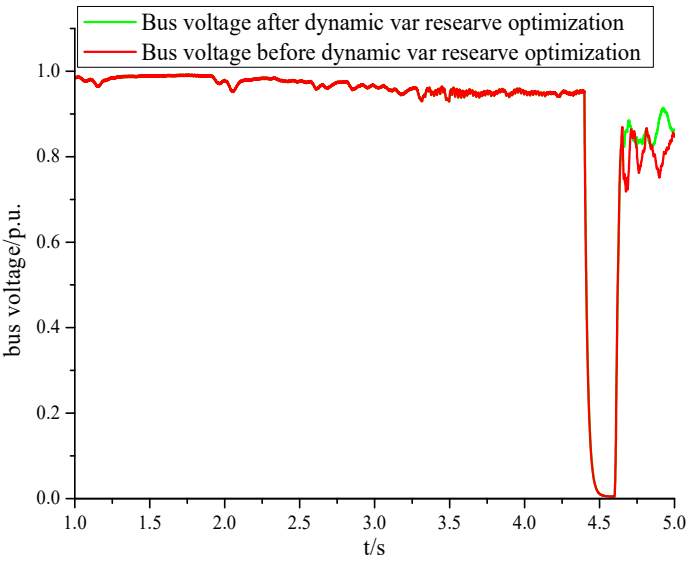

(d) three-phase grounding fault

Figure 7. Effectiveness and feasibility of the proposed method.

On the other hand, extinction angle is the criterion to judge whether commutation failure occurs in LCC-HVDCs system. If the extinction angle is too small, commutation failure always occurs. As shown in Figure 7b, before dynamic var reserve is configured, continuous commutation failure occurs in the system; after dynamic var reserve optimization, continuous commutation failure is effectively restrained, and the stability and safe operation level of the system are improved.

Table 3 shows the comparison between the standard answers obtained by solving TSCOPF and the results obtained by the methods presented in this paper. Obviously, the method proposed in this paper is simple to calculate, and accurate dynamic var reserve value can be obtained without solving the complex TSCOPF problem. Three-phase and single-phase grounding faults are set at the AC side respectively. The detailed fault information is referred to in Table 3. It also can be seen from Table 4 that after the installation of dynamic var equipment, the power loss of the system is significantly reduced.

Table 4. Comparative chart of electrical losses.

\begin{tabular}{ccc}
\hline \multirow{2}{*}{ Type of Faults } & \multicolumn{2}{c}{ Power Loss/MW } \\
\cline { 2 - 3 } & With SCs & Without SCs \\
\hline Single phase ground fault & 24.73 & 37.12 \\
Three phase grounding fault & 42.67 & 64.33 \\
\hline
\end{tabular}

On the other hand, due to the inclusion of new energy and electronically processed loads, the potential operation risk of hybrid AC/DC power grid is also increasing. Since the main research object of this paper is receiving end with multi-infeed LCC-HVDCs, 
this paper analyzes the change of voltage amplitude at load side in a non-sinusoidal environment to illustrate the feasibility of the method proposed in this paper. In this section, a single-phase grounding fault is set at the load side. The fault occurrence time is $0.04 \mathrm{~s}$ and the fault removal time is $0.06 \mathrm{~s}$. The detailed fault information is shown in Table 3. From the simulation results shown in Figure 8, it can be seen that with dynamic var equipment, voltage amplitude at load side can quickly recover to the three-phase symmetrical state. When the load has high requirements for power quality, the installation of dynamic var equipment can ensure that the load voltage can quickly recover to normal state after failure.

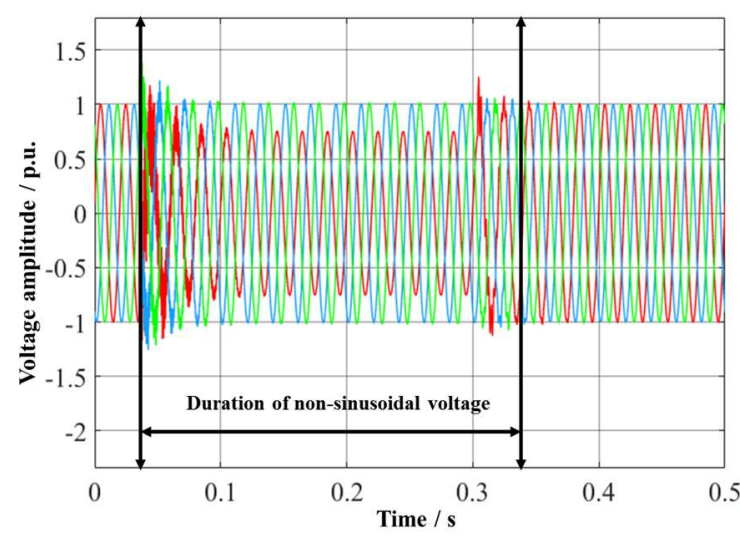

(a) Voltage waveform at load side without SCs

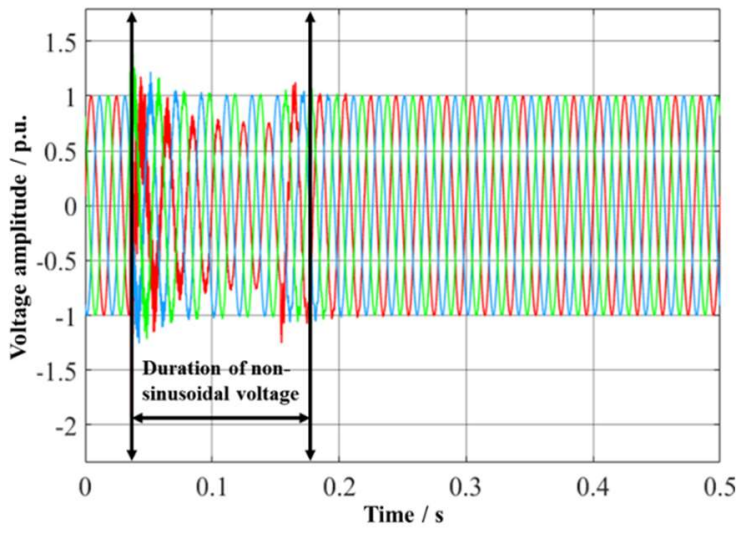

(b) Voltage waveform at load side with SCs

Figure 8. Comparison of three-phase non-sinusoidal duration of load voltage.

\subsection{Determination and Analysis of Voltage Security Region}

In order to further illustrate the effect of dynamic var equipment on voltage security and stability of power system, the voltage security region is obtained. The topology of modified IEEE-9 test system with external double-infeed LCC-HVDCs is shown in Figure 9. The converter stations at the receiving end are distributed on node 10 and 11, and are equipped with two synchronous condensers. The capacity of synchronous condenser is 20 MVAR and the var output range in steady state is set as [-10 MVAR, 20 MVAR].

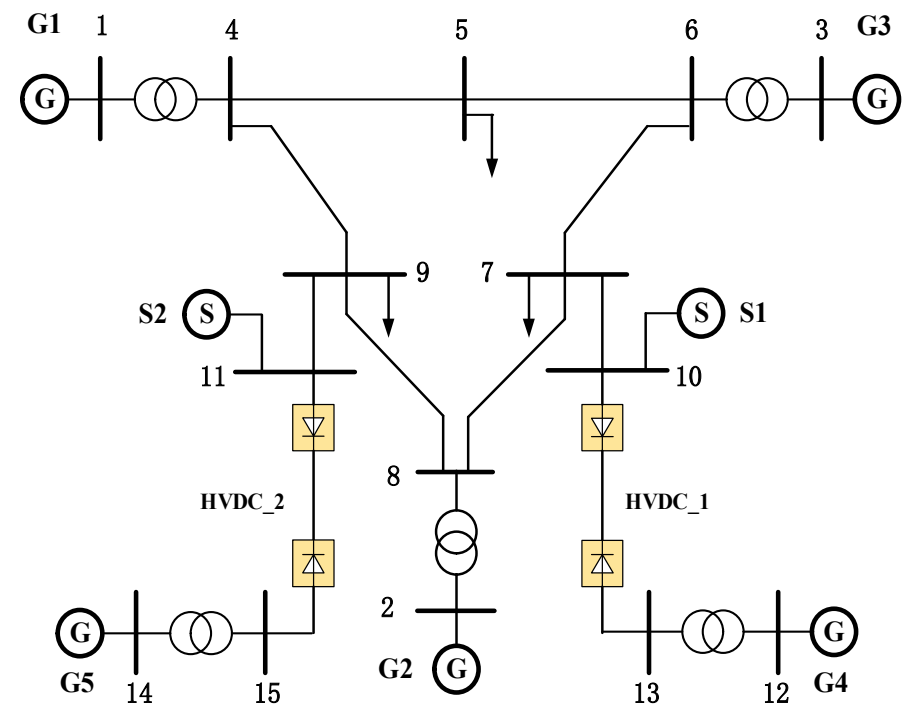

Figure 9. The topology of modified IEEE-9 test system.

Firstly, by Monte Carlo simulation, 1000 operation points are randomly generated and verified by $\mathrm{N}-1$ check. The unsafe operation points of voltage instability are represented by 
red area; the safe operation points satisfying all constraints under all faults are represented by green area, and the operation points with dynamic var equipment configuration in advance according to the method proposed in this paper are represented by the blue area. Because the dimension of voltage security region is high, for convenience of explanation, the voltage safety region is projected onto a two-dimensional plane for observation in this paper.

As shown in Figure 10, after installing synchronous condensers with enough capacity, the voltage security level is significantly improved and DC blocking fault is prevented effectively. Some advantages can be summarized.
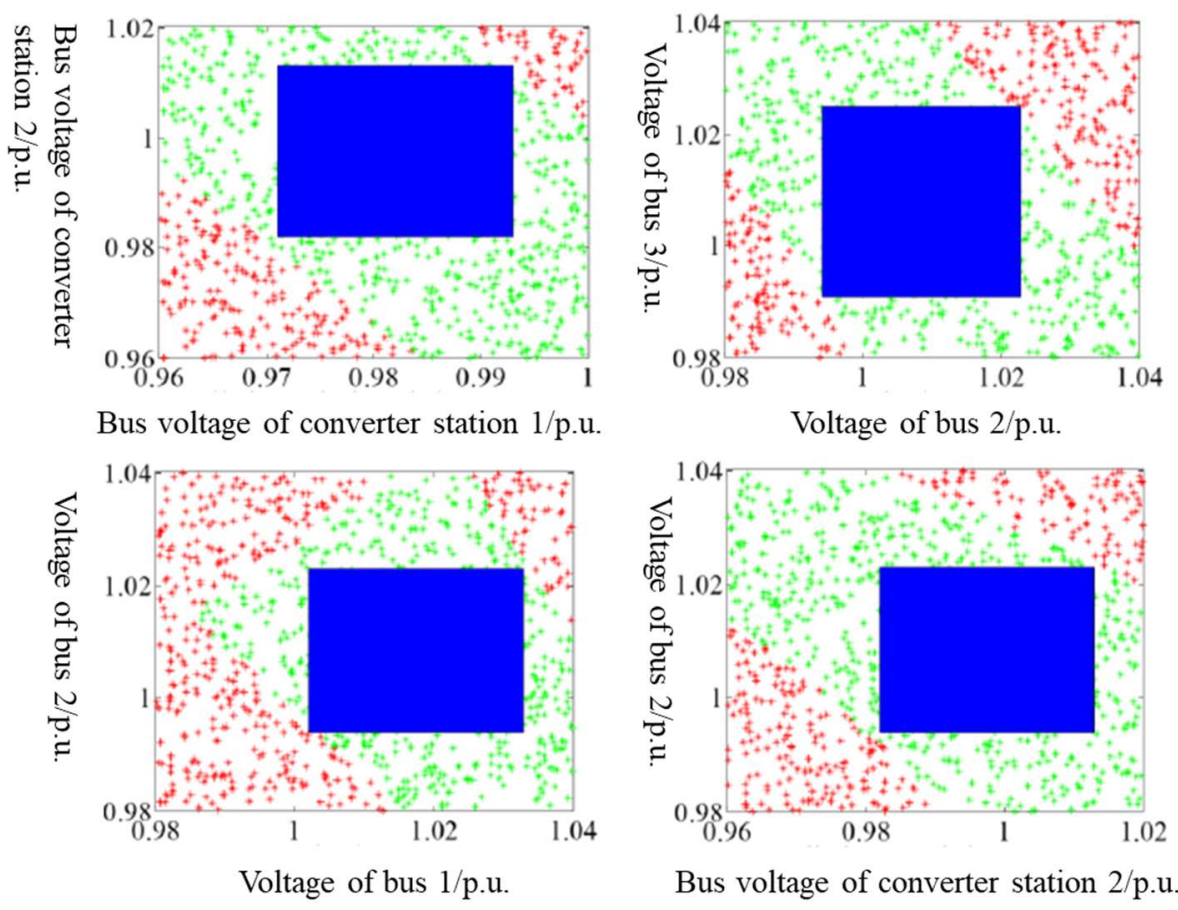

Figure 10. 2-dimensional plane projection of voltage security region.

(1) Continuity and flexibility of voltage regulation using dynamic var equipment: dynamic var equipment has the ability of continuous regulation, but traditional static var compensation equipment, such as capacitors, can only compensate with fixed capacity, which greatly limits the flexibility of var regulation resources. In addition, the limited var regulation capacity of capacitor bank will lead to some uncontrollable blank areas in the voltage security region. When the capacity of capacitors is small, the voltage security region of the system is still a continuous rectangle without blank areas; when the capacity of capacitors is too large, the voltage security region is no longer an intact region, as shown in Figure 11, but a union of several continuous regions. This shows that because the capacity of capacitors is too large, a large voltage regulation range will be brought by switching capacitors. No matter what kind of adjustment combination of generator and capacitor is adopted, the bus voltage of two substations cannot be adjusted to these blank areas. 


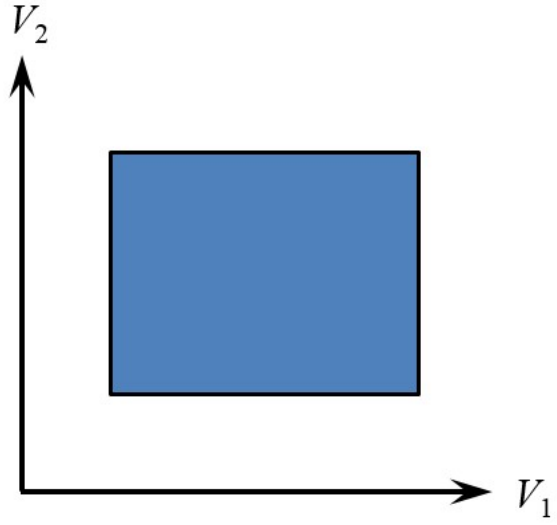

(a) The capacity of capacitor is too suitable

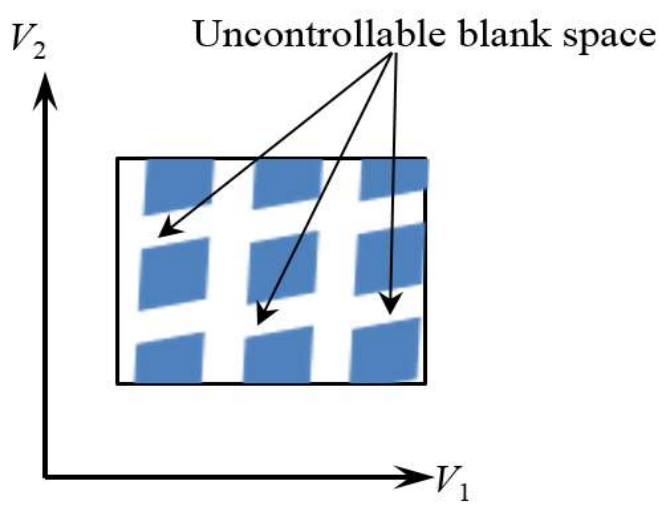

(b) The capacity of capacitor is too large

Figure 11. Voltage security region under different capacity of capacitor.

(2) Safety and economy of voltage regulation using dynamic var equipment: When the capacity of dynamic var equipment is too small, it cannot provide enough dynamic var support during failure period, even resulting in voltage collapse of the system. On the other hand, when the capacity of dynamic var equipment is too large, considering the high cost of dynamic var equipment, it will lead to increased investment and waste of resources. As shown in Figure 10, some vertexes of the voltage security region are always pressed on the boundary, which shows that the dynamic var reserve capacity obtained by the proposed method can just meet the requirements of the safe operation of the system. It should be noted that some vertexes are not on the boundary, because the voltage security region is a high-dimensional space. When they are projected onto the two-dimensional plane, the vertexes are no longer on the boundary.

\section{Conclusions and Future Work}

In order to overcome the shortcomings of high computational complexity and low solution accuracy of traditional dynamic var reserve evaluation methods, in this paper, a novel method to obtain dynamic var reserve capacity of SCs in multi-infeed LCC-HVDCs is proposed. This method does not need to solve the complex TSCOPF problem with the help of data fitting. Through data fitting method and improved Powell algorithm, the relationship between extinguishing angle of converter station and many other electrical parameters such as active power, reactive power and dynamic var equipment capacity can be described by high-order polynomials. The improved Powell algorithm can effectively overcome the limitations of the traditional least squares method and avoid falling into the local minimum value. Finally, the reserve capacity of dynamic var equipment is determined by step searching algorithm. In the part of case study, two test systems including double infeed LCC-HVDC system and the modified IEEE-9 system, are used to illustrate the effectiveness and feasibility of the proposed method. The following conclusions can be drawn from the simulation results:

1. After equipped with dynamic var equipment, the safe operation margin of hybrid AC/DC power grid is significantly improved. When faults occur in AC system, dynamic var equipment can quickly provide var and voltage support and prevent the further spread of fault influence.

2. It can be seen from the simulation results of voltage safety region that compared with traditional static var equipment, the dynamic var equipment can continuously adjust the voltage and enlarge the range of safe operation.

Although the research work of this paper mainly focuses on the dispatching operation of power system, the relevant methods and conclusions can be extended to system planning application. For power systems with multi-infeed LCC-HVDCs, how to equip appropriate dynamic var reserve for areas with serious voltage security and stability problems, includ- 
ing the capacity of configured equipment, the selection of installing address, the selection of equipment parameters and so on, can be deeply studied by using the conclusions given in this paper.

From the perspective of system control, this paper focuses on using the existing var and voltage regulation resources to make the system operate as safely as possible. However, with the expansion of the scale of China's power grid and the increasingly complex operation mode, the participation and regulation of the power market will become an important means in the future. In the scenarios of large-scale new energy, it will become the trend of future research to study the pricing strategy of reactive auxiliary services based on effective dynamic var reserve evaluation and provide new var and voltage regulation means for these areas with insufficient dynamic var reserve. The pricing strategy of var auxiliary service can also be deeply studied based on the relevant theories of dynamic var reserve evaluation and optimization proposed in this paper.

Author Contributions: Formal analysis, X.L. and L.F.; investigation, F.L.; methodology, L.T. and B.L.; resources, H.Y.; supervision, T.N. All authors have read and agreed to the published version of the manuscript.

Funding: This research is funded in part by the 2021 Science and Technology Project $1 \#$ of State Grid Chongqing Electric Power Company (Project No. SGCQJY00GHJS2100031), the National Natural Science Foundation of China (Project No. 52007017), Fundamental Research Funds for the Central Universities (Project No. 2020CDJQY-A027).

Institutional Review Board Statement: This study did not involve humans or animals.

Informed Consent Statement: Not applicable.

Data Availability Statement: Not applicable.

Conflicts of Interest: The authors declare no conflict of interest.

\section{References}

1. Hong, L.; Zhou, X.; Xia, H.; Liu, Y.; Luo, A. Mechanism and Prevention of Commutation Failure in LCC-HVDC Caused by Sending End AC Faults. IEEE Trans. Power Deliv. 2021, 36, 473-476. [CrossRef]

2. Xiao, H.; Li, Y.; Lan, T. Sending End AC Faults can Cause Commutation Failure in LCC-HVDC Inverters. IEEE Trans. Power Deliv. 2020, 35, 2554-2557. [CrossRef]

3. Wei, Z.; Yuan, Y.; Lei, X.; Wang, H.; Sun, G.; Sun, Y. Direct-Current Predictive Control Strategy for Inhibiting Commutation Failure in HVDC Converter. IEEE Trans. Power Syst. 2014, 29, 2409-2417. [CrossRef]

4. Xue, Y.; Zhang, X.; Yang, C. AC Filterless Flexible LCC HVDC With Reduced Voltage Rating of Controllable Capacitors. IEEE Trans. Power Syst. 2018, 33, 5507-5518. [CrossRef]

5. Lei, Y.; Li, T.; Tang, Q.; Wang, Y.; Yuan, C.; Yang, X.; Liu, Y. Comparison of UPFC, SVC and STATCOM in Improving Commutation Failure Immunity of LCC-HVDC Systems. IEEE Access 2020, 8, 135298-135307. [CrossRef]

6. Jacobson, D.A.N.; Wang, P.; Mohaddes, M.; Rashwan, M.; Ostash, R. A preliminary look at the feasibility of VSC HVDC in Manitoba. In Proceedings of the 2011 IEEE Electrical Power and Energy Conference, Winnipeg, MB, Canada, 3-5 October 2011; pp. 80-85.

7. Yang, T.; Yu, Y. Static Voltage Security Region-Based Coordinated Voltage Control in Smart Distribution Grids. IEEE Trans. Smart Grid 2018, 9, 5494-5502. [CrossRef]

8. Niu, T.; Guo, Q.; Sun, H.; Wu, Q.; Zhang, B.; Ding, T. Autonomous Voltage Security Regions to Prevent Cascading Trip Faults in Wind Turbine Generators. IEEE Trans. Sustain. Energy 2016, 7, 1306-1316. [CrossRef]

9. Li, G.; Zhang, S.; Jiang, T.; Chen, H.; Li, X. A method of detecting commutation failure in multi-infeed HVDC systems based on critical failure impedance boundary. In Proceedings of the 2017 IEEE Power \& Energy Society General Meeting, Chicago, IL, USA, 16-20 July 2017; pp. 1-5.

10. Rusek, S.; Střida, F.; Mach, M. The problems of electrical distance in connection with the operation of wind power generator. In Proceedings of the 2010 9th International Conference on Environment and Electrical Engineering, Prague, Czech Republic, 16-19 May 2010; pp. 65-68.

11. Gao, R.; Xu, Y.; Bao, L.; Cao, Z. Analysis of the electric railway loading impact on the operation of the generator negative current protection. In Proceedings of the 2008 IEEE Canada Electric Power Conference, Vancouver, BC, Canada, 6-7 October 2008; pp. 1-6.

12. Huang, Q.; Vittal, V. Application of Electromagnetic Transient-Transient Stability Hybrid Simulation to FIDVR Study. IEEE Trans. Power Syst. 2016, 31, 2634-2646. [CrossRef] 
13. Han, X.; Zhang, H. Power system electromagnetic transient and electromechanical transient hybrid simulation based on PSCAD. In Proceedings of the 2015 5th International Conference on Electric Utility Deregulation and Restructuring and Power Technologies (DRPT), Changsha, China, 26-29 November 2015.

14. Hu, B.; Niu, T.; Li, F.; Xie, K.; Li, W.; Jin, H. Dynamic Var Reserve Assessment in Multi-Infeed LCC-HVDC Networks. IEEE Trans. Power Syst. 2021, 36, 68-80. [CrossRef]

15. Geng, G.; Jiang, Q. A Two-Level Parallel Decomposition Approach for Transient Stability Constrained Optimal Power Flow. IEEE Trans. Power Syst. 2012, 27, 2063-2073. [CrossRef]

16. Pizano-Martinez, A.; Fuerte-Esquivel, C.R.; Ruiz-Vega, D. A New Practical Approach to Transient Stability-Constrained Optimal Power Flow. IEEE Trans. Power Syst. 2011, 26, 1686-1696. [CrossRef]

17. Xia, S.; Luo, X.; Chan, K.W.; Zhou, M.; Li, G. Probabilistic Transient Stability Constrained Optimal Power Flow for Power Systems with Multiple Correlated Uncertain Wind Generations. IEEE Trans. Sustain. Energy 2016, 7, 1133-1144. [CrossRef]

18. Geng, G.; Jiang, Q.; Sun, Y. Parallel Transient Stability-Constrained Optimal Power Flow Using GPU as Coprocessor. IEEE Trans. Smart Grid 2017, 8, 1436-1445. [CrossRef]

19. Liu, X.; Liu, Y.; Liu, J.; Xiang, Y.; Yuan, X. Optimal planning of AC-DC hybrid transmission and distributed energy resource system: Review and prospects. CSEE J. Power Energy Syst. 2019, 5, 409-422. [CrossRef]

20. Raza, A.; Liu, Y.; Rouzbehi, K.; Jamil, M.; Gilani, S.O.; Dianguo, X.; Williams, B. Power Dispatch and Voltage Control in Multiterminal HVDC Systems: A Flexible Approach. IEEE Access 2017, 5, 24608-24616. [CrossRef]

21. Zhou, M.; Zhai, J.; Li, G.; Ren, J. Distributed Dispatch Approach for Bulk AC/DC Hybrid Systems with High Wind Power Penetration. IEEE Trans. Power Syst. 2018, 33, 3325-3336. [CrossRef]

22. Huang, J.; Gao, H.; Zhao, L.; Feng, Y. Instantaneous Active Power Integral Differential Protection for Hybrid AC/DC Transmission Systems Based on Fault Variation Component. IEEE Trans. Power Deliv. 2020, 35, 2791-2799. [CrossRef] 